\section{Experiencia de integración de docencia y extensión universitaria para la ampliación de derechos de la niñez en comunidad}

\author{
Marcela D’Angelo \\ marsantafe@hotmail.com \\ Florencia Donayo \\ florenciadonayo@yahoo.com.ar \\ Verónica Heinrich \\ veronica_heinrich@yahoo.com \\ Docentes de la Escuela Superior de \\ Sanidad "Dr. Ramón Carrillo" de la \\ Facultad de Bioquímica y Ciencias \\ Biológicas. Universidad Nacional del \\ Litoral, Argentina.
}

Terapeuta Ocupacional del Centro de Atención Primaria Intercultural Nalequetequeta", de la ciudad de Recreo, Santa Fe, Argentina. lore_ramos78@hotmail.com
Integración de la docencia y la extensión /

Intervenciones

RECEPCIÓN: 24/06/16

ACEPTACIÓN FINAL: 10/10/16

\section{Resumen}

El presente trabajo comparte la experiencia de tejer territorio con tramas conjuntas de actores locales de la ciudad de Recreo y de la Universidad Nacional de Litoral. Partimos del diagnóstico situacional construido con la comunidad, donde niños y niñas expresan sus inquietudes de ampliar y disponer de recursos y/o espacios de ocupaciones significativas lúdicos y recreativos; siendo un aporte el Proyecto de Educación Experiencial: Inquietes ${ }^{1}$ Derechos en Movimiento, en acompañar y contribuir intersectorialmente a procesos de participación protagónica de la niñez en esta localidad en todos los momentos metodológicos y a la formación universitaria de estudiantes promotores de salud. Se destaca que enseñar y aprender con los movimientos de salud de la propia comunidad permite el reconocimiento colectivo acerca de la importancia de contar con otro/a a la hora de abordar situaciones en su complejidad desde el enfoque de derechos.
Palabras-clave

- Niñez

- Territorio

- Formación universitaria

- Intersectorialidad

- Ciudadanía

\section{Resumo}

O presente trabalho compartilha a experiência de entrelaçar território com tramas conjuntas de atores locais da cidade de Recreo e da Universidade Nacional del Litoral. Começamos com o diagnóstico da situação construída com a comunidade, onde as crianças expressaram suas preocupações de ampliar e dispor de recursos e/ou espaços significativos lúdicos e de recreação; sendo uma contribuição ao projeto de Educação Experiencial:Inquietos, Direitos em Movimento, acompanhar e contribuir intersetoralmente para os processos de participação ativa das crianças nesta cidade em todos os momentos metodológicos e para a formação dos estudantes universitários promotores de saúde. Destaca-se que ensinar e aprender com os movimentos de saúde da própria comunidade permite o reconhecimento coletivo da importância de ter mais alguém na hora de tratar situações complexas a partir da abordagem dos direitos.

Palavras-chave

- Infância

- Território

- Formação universitária

- Intersetorialidade

- Cidadania
Para citación de este artículo

D’Angelo, M; Donayo, F.; Heinrich, V. y Ramos, L. (2016).

Experiencia de integración de Docencia y Extensión universitaria para la ampliación de derechos de la niñez en comunidad. En Revista +E versión digital, (6), pp. 376-381. Santa Fe, Argentina: Ediciones UNL. 


\section{Introducción}

"(...) el significar el rol como facilitadoras de salud, el participar 'participando' y el jugar 'jugando', considerando que el juego es la forma de participación del niño, que son gestores del proyecto, al concretarse los espacios lúdicos en el patio pudieron expresarse, relacionarse con otros pares, tomar decisiones acerca del juego, elegirlos, modificar las reglas, esperar su turno, respetar al compañero que está jugando, vivenciando valores, aprendiendo y divirtiéndose; contribuyendo a la construcción de su ciudadanía a través del ejercicio de sus derechos". (Gallo, Maggio, 2015) $)^{2}$

El presente trabajo pretende compartir nuestras reflexiones respecto al camino recorrido en las búsquedas profesionales y colectivas de transformación de situaciones sociales de las cuales somos parte y que se enlazan con distintos territorios de enseñanzas y aprendizajes a las cuales nos sumamos en la identificación y construcción de oportunidades concretas en el acceso y ejercicio de derechos en la niñez.

Este siendo parte del contexto desafía a la búsqueda de estrategias desde el equipo universitario que procura ampliar la mirada e integrar los recursos conceptuales, metodológicos y técnicos a fin de acompañar procesos comunitarios implicándonos en la cotidianeidad de niños y niñas "la cual se funda en retomar en forma histórica y contextualizada al sujeto y su inserción participante en el colectivo" (Galheigo, 2003:108). Desde allí implica tejer territorio entendido como "espacio producido socialmente, sea por medio de prácticas espaciales (espacio vivido), formas de representación, mapas, figuras (espacio representado) o a través de símbolos, ideas o conceptos (espacio simbólico)" (Manzanal, 2007:42) tanto en la Universidad como en la comunidad de Recreo. Este andar-andando se realiza desde el año 2012 hasta la fecha, y en él nos involucramos desde la cátedra de Práctica Profesional
Terapia Ocupacional (TO) en Comunidad ${ }^{3}$ en la ciudad de Recreo, provincia de Santa Fe, Argentina, en el Centro de Atención Primaria Intercultural ${ }^{4}$ de Salud (CAPS) "Natarentanca com Nalequetequeta". Ello nos ha permitido ir al encuentro de las voces de niños, niñas y referentes próximos (docentes, equipo de salud, estudiantes de la práctica profesional), quienes expresan sus deseos e inquietudes de ampliar y disponer de espacios de ocupaciones significativas y/o recursos materiales, físicos y simbólicos que permitan experimentar, explorar y vivenciar nuevas actividades recreativas y lúdicas en contextos propios de sus vidas cotidianas.

La aproximación diagnóstica participativa permitió advertir:

- Asunción temprana, por parte de los niños -y particularmente las niñas- de roles de cuidado de familia y tareas del hogar.

- Insuficientes posibilidades de acceder a propuestas artísticas, recreativas, deportivas de acuerdo con intereses, creencias y valores.

- Limitadas opciones de participación por fuera de la escuela y de la familia.

- Escasas oportunidades de expresar y desarrollar su identidad cultural. $^{5}$

- Presencia de barreras epistémicas, subjetivas y políticas que dificultan las oportunidades de participación ocupacional de niños, niñas y jóvenes en la comunidad. ${ }^{6}$

Es así que se motorizan distintas acciones colectivas tanto en el espacio público como en las dinámicas escolares donde ellos y ellas transitan, procurando durante el proceso comunitario favorecer la formación de estudiantes universitarios como agentes de promoción de salud en pos de contribuir con la construcción de ciudadanía en la niñez. Enlazar las políticas universitarias a los movimientos de salud en la comunidad desde un enfoque de derechos supone imprimir a las acciones los atributos de
1) Inquietes se nombra con "e" para incluir las diversidades genéricas. 2) Corresponde al Trabajo Final Integrador realizado por estudiantes de Terapia Ocupacional luego de participar del PEE

3) De la Licenciatura de Terapia Ocupacional de la Escuela Superior de Sanidad "Dr. Ramón Carrillo",

Plan de Estudio 2014, ubicada en el $5^{\circ}$ año. Validez Nacional por Res. $n^{\circ}$ 1811/1998 del Ministerio de Cultura y Educación. Facultad de Bioquímica y Ciencias Biológicas. Universidad Nacional del Litoral. Santa Fe. Argentina 4) La nominación Intercultural deviene porque su área de responsabilidad abarca tanto población criolla como de la etnia mocoví. Estos últimos son originarios del Gran Chaco (Argentina), fueron sociedades cazadoras-recolectoras organizadas como unidades o "bandas" familiares, que sufrieron tempranamente la colonización y evangelización española lo cual implicó grandes cambios en sus sociedades con una progresiva asimilación a la población criolla de la zona no sin resistencia o rebelión por parte del pueblo mocoví. Fueron desplazados hacia el sur de la provincia del Chaco y norte de la provincia de Santa Fe, desde donde llegaron a la ciudad de Recreo en el año 1968 para trabajar como peones rurales.

5) Este aspecto hace especial referencia a la comunidad mocoví donde la segregación cultural y social genera el ocultamiento de su identidad de origen. En las últimas décadas se han visibilizado los pueblos originarios a través de sus luchas por derechos ciudadanos y puntualmente en el barrio mocoví con la construcción de Centro de Salud intercultural, Escuela primaria intercultural.

6) Maffia (2005), en torno a las barreras que dificultan el acceso y ejercicio de derechos define las siguientes categorías conceptuales: Barrera epistémica: tiene que ver con el conocimiento y consisten en que muchas veces las personas no conocen sus derechos. Barrera subjetiva: aunque las personas conozcan sus derechos no suelen sentirse merecedoras o que les corresponda por el solo hecho de ser humano. Barrera política: tiene que ver con la falta de recursos materiales, humano y de comunicación para hacer valer las normas. Las leyes no son condición suficiente para que un derecho valga. Tiene que ver con voluntad política que debe materializarse. 
universalidad, la exigibilidad, la participación social, la integralidad y la progresividad, en tanto este enfoque, según Pautassi, consiste en:

"el amplio conjunto de principios, reglas y estándares que integran los derechos humanos fundamentales, y que son pasibles de ser aplicados para fijar pautas y criterios para el diseño e implementación de estrategias de desarrollo sustentable y con mayor interés aún, en materia de políticas sociales". (2010:2)

Se establecen ejes de acción claves que se enlazan mutuamente y posibilitan ampliar, en proceso, las oportunidades de juego y recreación para la niñez entendiendo que es un aporte fundamental a la mejora de su calidad de vida e inclusión social. Estos ejes de acción son:

- Procurar la participación de niños, niñas y grupos familiares en los distintos escenarios de la vida cotidiana: hogar, escuela, CAPS, espacios públicos y asociaciones civiles, promoviendo las decisiones, elecciones, exploración, expresión y creatividad en cada actividad, taller, encuentro.

- Considerar al CAPS como referente territorial estratégico dado por su visión institucional y su metodología de abordajes integrales hacia y con la población tanto criolla como mocoví, posibilitando la implementación de las acciones y articulaciones con las distintas instituciones.

- Considerar a la comunidad educativa como referente significativo, acompañar la crianza y educación de la niñez como un espacio de socialización, inclusión y promotor de salud que puede propiciar el ensamble de saberes populares, académicos, interculturales.

- Planificar acciones desde las perspectivas de género, derechos e interculturalidad, que tensionen las cosmovisiones dominantes, estereotipos y mitos que refuerzan los prejuicios y la discriminación e impactan sobre la subjetividad de las personas y la convivencia en comunidad.

- Implementar en cada proyecto el enfoque participativo dando lugar a la voz de actores en todas las fases del mismo: aproximación diagnóstica, implementación y monitoreo como modo de construcción de ciudadanía en la niñez visibilizando las capacidades y potencialidades de la comunidad: empoderamiento.

\section{Entramando territorios}

Surge así el proyecto "Inquietes: Derechos en movimiento", diseñado desde un enfoque participativo e intersectorial en el año 2014, presentado y aprobado bajo Resolución de CD FBCB

7) Instituciones participantes universitarios: Universidad Nacional del Litora (UNL): Facultad Bioquímica y Ciencias Biológicas (FBCB) Licenciatura en Terapia Ocupacional. Cátedras: Socio- logía y Práctica Profesional "Terapia ocupacional en comunidad". Facultad de Arquitectura, Diseño y Urbanismo (FADU) Licenciatura en Diseño de la Comunicación Visual (LDCV): Cátedra $n^{\circ} 167$ en la convocatoria institucionalizada de la Secretaría de Extensión de UNL a la modalidad de Prácticas de Educación Experiencial, y desarrollado desde febrero a diciembre de $2015^{7}$ con la creencia de que las multidimensiones de la cuestión social y la creciente inquietud de pensar propuestas integrales para su abordaje a través de la articulación entre distintas cátedras pertenecientes a distintos claustros universitarios propicia la construcción de miradas integrales y la oportunidad de acoplar saberes académicos a las políticas de salud, educación, y desarrollo social que impactan a nivel local. Cunill Grau, citando a Junqueira, refiere a la intersectorialidad como la

"articulación de saberes y experiencias en el planeamiento, realización y evaluación de acciones, con el objetivo de alcanzar resultados integrados en situaciones complejas, buscando un efecto sinérgico en el desarrollo social" (Junqueira y otros, 1998:41).

El Proyecto pretende ensamblar diferentes actores y territorios desde una perspectiva intersectorial, que en la tarea de mejorar la calidad de vida de niños y niñas favorece la participación ocupacional desde un enfoque de derechos, integra a la Universidad, el CAPS, las escuelas y la Municipalidad de Recreo. Para ello se propone como estrategia metodológica sostener durante todo el proceso reuniones sistemáticas quincenales donde convergen y participan referentes institucionales y comunitarios: estudiantes de diferentes carreras, trabajadores del CAPS, docentes de las escuelas, funcionarios y trabajadores municipales, miembros de la comunidad para monitorear, planificar y direccionar las acciones del Proyecto. Conforme a Manzanal:

"Para un encuadre teórico-metodológico como el que proponemos necesitamos enfocar, asimismo, en los actores y sujetos del territorio en cuestión. Porque debemos dar cuenta de las potencialidades y capacidades presentes en los propios actores y sujetos para intervenir y modificar su realidad.

Porque precisamos informar sobre los sentidos y modalidades que ellos tienen y aplican para transformar la desigualdad en la que están inmersos". (2007:22)

\section{Tramas en el territorio local: acciones del Proyecto} "Inquietes" en ámbitos comunitarios de la ciudad de Recreo El CAPS es un centro de referencia local para otras instituciones ubicadas en la comunidad de Recreo, lo que permite sostener y profundizar lazos entre los actores locales, estudiantes y profesionales que participan del proyecto y posibilitar la
Morfología 1.Instituciones localidad de Recreo: Centro Atención Primaria Salud (CAPS) intercultural Natarentanca com Nalequetequeta Escuela Primaria Intercultural nro. 1338 Com Caia.
Escuela Primaria $n^{\circ} 1277$ Robustiano Aldao. Municipalidad de Recreo. Provincia de Santa Fe, Argentina. 


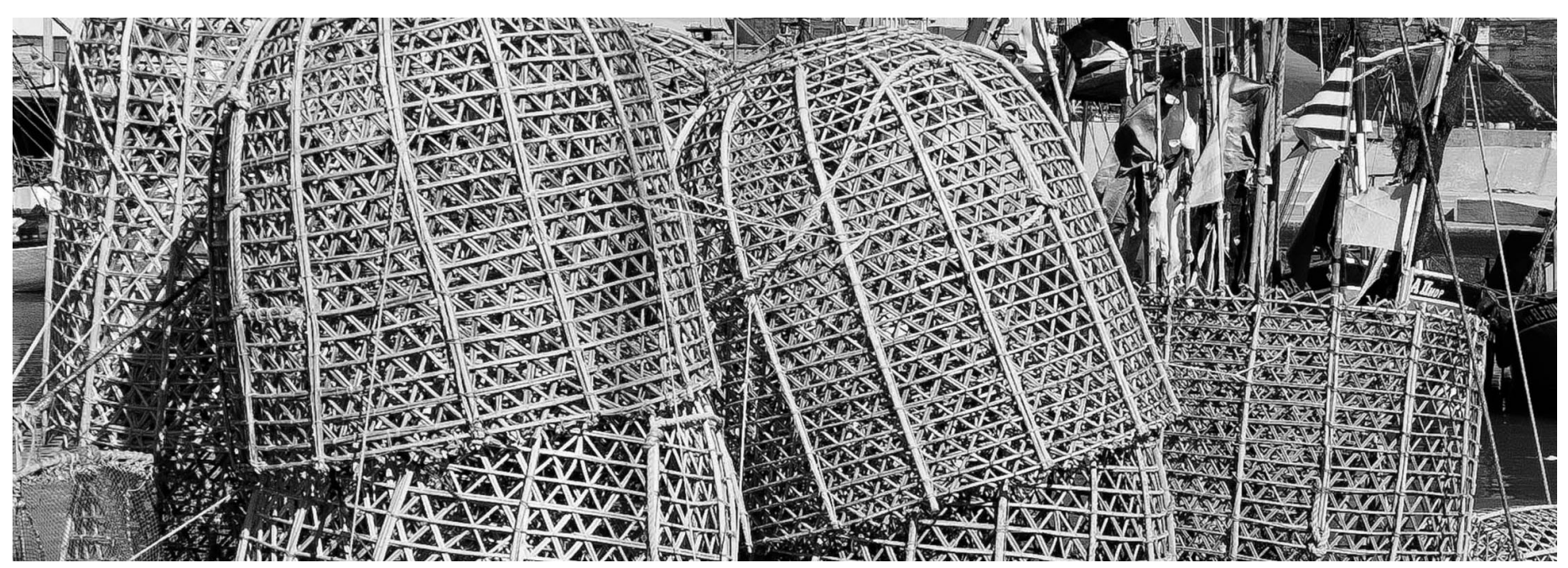

(c) Gastón Pignata

implementación de políticas de salud desde una perspectiva integral y territorial situada, en un proceso de retroalimentación y articulación permanente entre la singularidad de cada anclaje territorial y la relectura de la formación teórico-instrumental necesaria para definir las nuevas coordenadas que cada situación exige, y desde lo que cada nueva situación enseña en la vivencia. Es desde este espacio institucional que se diseñan, planifican e implementan participativamente talleres de expresión cultural, denominados por los propios niños y niñas "Talleres de Diversión", a desarrollarse en los barrios Mocoví y Comunal. Se exploran expectativas, intereses y propuestas en torno a la intervención en el espacio público y se desarrollan actividades lúdicas, artísticas y recreativas coordinadas por estudiantes universitarios de TO y de otras carreras (Licenciatura de Trabajo Social y Licenciatura en Música)

Por otra parte, en las escuelas se organizan talleres semanales lúdicos y expresivos y se planifican proyectos consecuentes con el diagnóstico participativo que en cada espacio se construye. En la Escuela $n^{\circ} 1277$ Robustiano Aldao emerge desde las voces de los niños y niñas una propuesta para abordar el horario del recreo como oportunidad de encuentro y convivencia, y surge así con alumnos y alumnas de $6^{\circ}$ y $7^{\circ}$ grado el Proyecto Colectivo "Sueña tu recreo", Intervención en el patio de la escuela, por la cual se pintan juegos elegidos con la participación de quienes asisten al nivel primario en sus distintos grados (6 años a 13 inclusive). En la inauguración del patio con oportunidades de juego aparece un recreo con otro matiz, respecto de lo cual las estudiantes universitarias expresan:

"Al tocar el timbre los niños/as se acercan corriendo a los juegos demostrando alegría y emoción, el patio se vistió de fiesta y color, ya no se ven niños/as dispersos por todos lados, sino concentrados en varios espacios del mismo, jugando y divirtiéndose". (Gallo y Maggio, 2015:8)

En la Escuela Intercultural $n^{\circ} 1338$ Com Caia se construyeron juegos de mesa y otros para los patios y espacio verde con la participación de alumnos, alumnas, docentes de la escuela y estudiantes de TO, quienes reflexionan al concluir su práctica y dicen:

"El TO participando en políticas sociales participativas requiere emplear estrategias de intervención que pasen por el fortalecimiento de los sujetos y las comunidades para revertir la exclusión e iniciar un proceso de restitución de derechos".

(Bertoncello y Suarez 2016:11)

Progresivamente, en los distintos encuentros se va dando cuerpo a la idea de transformar el espacio público lindante al CAPS en una plaza a partir de las expresiones de niños y niñas que muestran deseos de disponer de juegos en donde se pueda:

"trepar, volar, saltar, correr, pintar, empujar, subir, bajar, escalar (...). Imaginan tener SU plaza ideal con pasamanos para trepar, hamacas, toboganes, subibajas, ta-te-ti para plaza, arcos de futbol, aros de básquet, laberintos, túneles, telarañas para trepar, rayuelas, lugar para pintar, instrumentos musicales". 8 (Proyecto "Inquietes", monitoreo: 1)

8) Documento interno equipo de Extensión Inquietes. UNL, Informe de monitoreo, 2016, p. 2. 


\section{4}

llevar adelante metodologías

participativas en la construcción

de un proyecto social que sea

eco de las voces de actores

diversos implica, en la formación

de estudiantes universitarios, poner el acento en enseñar a

"aprender a escuchar"
Es así que las voces y diseños realizados desde la niñez se van ensamblando con aportes que desde la cátedra de Morfología, estudiantes y equipo docente de la Licenciatura en Diseño y Comunicación Audiovisual llevan a cabo en instancias analítica, exploratoria y de reconocimiento del emplazamiento situacionalsocial, trabajando in situ en el área del CAPS —Recreo y luego en la Facultad de Arquitectura, Diseño y Urbanismo (FADUUNL) - una instancia de tarea compartida y de intercambio entre estudiantes, docentes de las distintas cátedras y escuelas junto a niños y niñas de la comunidad.

La plaza inaugura su primera etapa el día 20 de noviembre de 2015 en consonancia con la conmemoración de la Convención Internacional de los Derechos de la Niñez como primer mojón de un trabajo colectivo que abarca desde el diseño del bosquejo de la plaza y muestra de los talleres realizados en el año hasta la realización de los primeros juegos, enlazando recursos, ideas, decisiones que implican movimientos en redes formales e informales a nivel operativo, técnico y político. La voz de niños y niñas se amplifica y logra definiciones con relación al uso del espacio público desde la propia intendencia (gobierno local).

\section{Tramas en el territorio académico: acciones del Proyecto "Inquietes" en ámbitos de la Ciudad Universitaria (FADU/FBCB/Predio UNL)}

Llevar adelante metodologías participativas en la construcción de un proyecto social que sea eco de las voces de actores diversos implica, en la formación de estudiantes universitarios, poner el acento en enseñar a "aprender a escuchar" e ir al encuentro de un "otro/otra" que pueda expresar auténticamente sus representaciones simbólicas, valores, creencias, prioridades, deseos, lo cual supone interpelar la posición hegemónica del profesional de la salud con supuesto poder — dado por el saber académico- para decidir sobre lo que es mejor en la vida de ese otro/otra. Es por eso que durante todo el proceso de enseñanzaaprendizaje valoramos la construcción de una mirada reflexiva y crítica que atraviese la

"intervención profesional, ya que entendemos que en ella subyacen supuestos epistemológicos, éticos, políticos, que pueden sostener opresiones o aportar a la autonomía de las personas y comunidades". (Cazzaniga, 1997:15)

Para ello, desde la Práctica Profesional de TO se sostienen instancias de supervisión con quienes en particular asisten diariamente a los espacios de encuentro pautados en la comunidad y se abordan en el aula a modo de seminarios semanales participativos. Los ejes temáticos que resultan prioritarios analizar al intervenir profesionalmente en el ámbito comunitario son: vida cotidiana, comunidad, alteridad, cultura, 
interculturalidad, desigualdad social, ciudadanía, salud, enfoques de derechos y género, participación, empoderamiento, redes. La metodología de trabajo que adoptamos es la de integrar la teoría con la vivencia, lo cual nos permite la comprensión a través de una reflexión consciente al considerar el aula también como un territorio en el cual, mediante dinámicas participativas, construimos comunidad, redes y equipo, recreamos contexto y cultura, percibimos desigualdades, vivimos la salud, autonomía y participación singular y colectiva. En palabras de Restrepo, se trata de utilizar:

'Los métodos pedagógicos de la 'educación popular', que refuerzan la confianza en sí mismo y la libre determinación, (los que) se aplican hoy de nuevo en gran medida en los campos de la educación y la comunicación en salud". (Restrepo, 2001:44)

Desde Sociología, los y las estudiantes participan de los seminarios introductorios realizados en las instalaciones de la FBCB y el predio UNL-ATE, pudiendo vivenciar conceptos como vida cotidiana, contextos, ciudadanía, participación, juego, derechos, relacionándolos con contenidos del plan de cátedra que tratan sobre el sentido común, la desnaturalización de lo social, la acción social y el rol de las instituciones, la interacción social, lo que posibilita la deconstrucción de los conocimientos a través de la participación en los diferentes seminarios y con los distintos actores en la comunidad. A la vez, el equipo docente de la Licenciatura de Diseño y Comunicación audiovisual, desde Morfología I, lleva a cabo en FADU (Taller 5) la instancia inicial y de puesta en contacto con el tema y sus derivaciones teóricas y la instancia resolutiva, comunicacional-espacial, en donde la experiencia y el contacto directo con tareas de campo en el medio físico, social y simbólico en la ciudad de Recreo y con la comunidad permite intercambios mutuos de saberes que definen la puesta a punto de la Muestra Inquietes Derechos en movimiento. En las evaluaciones de proceso los y las estudiantes destacan como aprendizajes: "el trabajo interdisciplinario, el diálogo y confianza con todo el personal del CAPS"; "el interés por conocer una cultura diferente a la propia"; "el diálogo y las dinámicas activas y participativas"; "la escucha y el respeto por los espacios y los otros"; "el conocimiento y el trabajo en el ámbito comunitario", "la toma de decisiones"; "la confianza que los niños brindaron, su capacidad para participar en el proyecto, aportando ideas, sugerencias, opiniones"; "el apoyo de los docentes durante el proceso, mostrando compromiso, responsabilidad y profesionalismo en todos los momentos del proceso", "bibliografía facilitada por la cátedra". 9

\section{Se hace trama al andar...}

Integrar extensión y docencia universitaria, entramar territorios, como venimos diciendo, es un camino con altibajos, curvas, rispideces, llanos y paisajes entrañables. Aprendemos todo el tiempo que los conflictos se van resolviendo en espacios colectivos de comunicación abierta y comprensiva que da lugar a las distintas voces y saberes. Que la confianza se construye con la presencia sostenida en tiempo, espacio y tarea. Que la participación de la comunidad con sus capacidades, deseos, necesidades y potencialidades es el eje que sostiene los vaivenes de los procesos sociales. Que es tan importante el aprendizaje de conocimientos y saberes teóricos en la formación de estudiantes universitarios/as como el desarrollo actitudinal de implicancia y sensibilidad social para trabajar creativamente con otros y otras de-construyendo en las vivencias aquello que aparece como verdad dada, absoluta y prescripta. Que las vivencias son en la comunidad, en el aula y en diálogo mutuo. Que desde la Universidad se aporta a ampliar derechos y salud cuando ésta se suma como un actor más que contribuye desde su particularidad en un proceso de acción colectiva que nos implica en un nosotros.

\section{Referencia Bibliográficas}

Bertoncello R.; Suarez M. (2016) Trabajo Final Integrador no publicado, UNL, Santa Fe, Argentina.

Buthler, J. (2009). "Lenguaje, poder e Identidad”, Ed. Síntesis. Madrid

Cazzaniga, S. (1997) "El abordaje desde la singularidad", Revista desde el fondo. Facultad de Trabajo Social. Universidad Nacional de Entre Ríos.22.

Cunill Grau, N. (2005). "La intersectorialidad en el gobierno y gestión de la política social". Congreso Internacional del CLAD sobre la reforma del Estado y de la Administación Pública.18,21. Santiago de Chile.

Galheigo, S. (2003). O cotidiano na terapia ocupacional: cultura, subjetividade e contexto histórico-social. Revista de Terapia Ocupacional da Universidade de São Paulo, 14 (3), 104-109. doi:http://dx.doi.org/10.11606/issn.2238-6149. v14i3p104-109.

Gallo G. y Maggio, A. (2015) "Repensando la intervención profesional como facilitadotas del proceso participativo en el proyecto escolar Sueña tu recreo". Trabajo Final Integrador no publicado, UNL, Santa Fe, Argentina.

Maffia, D. (2005) "Mujer y derecho". En cuaderno de proposiciones y prácticas. Año III. N 7. Edit. Acción Educativa. Santa Fe.

Manzanal, M.; Arzeno, M. y Nussbaumer, B. (2007) "Territorios en construcción: actores, tramas y gobiernos, entre la cooperación y el conflicto". 1a ed. - Buenos Aires Fundación Centro Integral Comunicación, Cultura y Sociedad - CICCUS. Pautassi, L. (2010). "El aporte del enfoque de Derechos a las políticas sociales. Una Breve revisión". CEPAL.Recuperado de http:///www.buenosaires.gob.ar/ areas/salud/dircap/mat/matbiblio/pautassi.pdf

Restrepo, H. E. de (2001). "Incremento de la capacidad comunitaria y del empoderamiento de las comunidades para promover la salud" Revista Facultad Nacional de Salud Pública. Universidad de Antioquia. 19. 1. Colombia. 